\title{
Application of the missing mass method in the fixed-target program of the STAR experiment ${ }^{*}$
}

\author{
Yuri Fisyak ${ }^{1}$, Victor Ivanov ${ }^{2,3}$, Hongwei $\mathrm{Ke}^{1}$, Ivan Kisel ${ }^{4,5,6,7}$, Pavel Kisel ${ }^{2,5, * *}$ Grigory \\ Kozlov $^{2,5}$, Spyridon Margetis ${ }^{8}$, Aihong Tang ${ }^{1}$, Iouri Vassiliev ${ }^{4}$, and Maksym Zyzak ${ }^{* * *}$ \\ (for the STAR Collaboration) \\ ${ }^{1}$ BNL, Brookhaven National Laboratory, Upton, NY, USA \\ ${ }^{2}$ JINR, Joint Institute for Nuclear Research, Dubna, Russian Federation \\ ${ }^{3} \mathrm{MEPhI}$, National Research Nuclear University, Moscow, Russian Federation \\ ${ }^{4}$ GSI, Helmholtz Center for Heavy Ion Research, Darmstadt, Germany \\ ${ }^{5}$ Goethe University, Frankfurt am Main, Germany \\ ${ }^{6}$ FIAS, Frankfurt Institute for Advanced Studies, Frankfurt am Main, Germany \\ ${ }^{7}$ HFHF, Helmholtz Research Academy Hesse, Frankfurt am Main, Germany \\ ${ }^{8} \mathrm{KSU}$, Kent State University, Kent, OH, USA
}

\begin{abstract}
The search for short-lived particles is usually the final stage in the chain of event reconstruction and precedes event selection when operating in online mode or physics analysis when operating in offline mode. Most often such short-lived particles are neutral and their search and reconstruction is carried out using their daughter charged particles resulting from their decay.

The use of the missing mass method makes it possible to find and analyze also decays of charged short-lived particles, when one of the daughter particles is neutral and is not registered in the detector system. One of the most known examples of such decays is the decay $\Sigma^{-} \rightarrow n \pi^{-}$.

In this paper, we discuss in detail the missing mass method, which was implemented as part of the KF Particle Finder package for the search and analysis of short-lived particles, and describe the use of the method in the STAR experiment (BNL, USA).

The method was used to search for pion $\left(\pi^{ \pm} \rightarrow \mu^{ \pm} v\right)$ and kaon $\left(K^{ \pm} \rightarrow \mu^{ \pm} v\right.$ and $K^{ \pm} \rightarrow \pi^{ \pm} \pi^{0}$ ) decays online on the HLT farm in the express production chain. An important feature of the express production chain in the STAR experiment is that it allows one to start calibration, production, and analysis of the data immediately after receiving them.

Here, the particular features and results of the real-time application of the method within the express processing of data obtained in the BES-II program at a beam energy of $3.85 \mathrm{GeV} / \mathrm{n}$ when working with a fixed target are presented and discussed.
\end{abstract}

\footnotetext{
*Work supported by BMBF and HFHF.

**e-mail: P.Kisel@gsi.de

***A Associated with FIAS.
} 


\section{Introduction}

The main goal of modern experiments with heavy ions, such as CBM (FAIR/GSI, Darmstadt, Germany), ALICE (CERN, Geneva, Switzerland) and STAR (BNL, Upton, USA), is a comprehensive study of the QCD phase diagram in the quark-gluon plasma (QGP) region and the possible phase transition from hadronic to QGP phase.

Strange particles are sensitive probes of the medium formed by heavy ion collisions. Since strange particles have decay modes with at least one neutral daughter particle, their decay products cannot be registered in the tracking detector system. To identify them, we proposed and implemented the missing mass method.

The method of missing mass was originally developed within the package FLES (First Level Event Selection) [1,2] for the CBM experiment. As part of the method development and research at CBM, the method showed its high efficiency on simulated data. However, it was important, especially for the first stage of the CBM experiment, to test on real data the concept of the developed approach and its current implementation. The FAIR Phase-0 program $[3,4]$, which includes participation in the STAR experiment at BNL (USA), gives us such an opportunity.

\section{Missing mass method}

The missing mass method is used to reconstruct decays of short-lived charged particles, when one of the daughter particles is neutral and is not registered in the detector system. The method is based on the laws of conservation of energy and momentum.

As an illustration of the method, let's consider a well-known decay

$$
\Sigma^{-} \rightarrow n \pi^{-} .
$$

In this decay, the daughter pion is registered in the tracking system and is identified in one of the PID detectors, while the neutron is not detected. However, the sigma particle, although it has a short lifetime $c \tau_{\Sigma^{-}}=4.4 \mathrm{~cm}$, but in fixed-target experiments due to relativistic boost the average length of trajectory in the laboratory system can reach $15-20 \mathrm{~cm}$. In this case the $\Sigma^{-}$particle will produce measurements in several tracking stations and can therefore be reconstructed. Thus, in the given decay, two charged tracks out of three can be reconstructed.
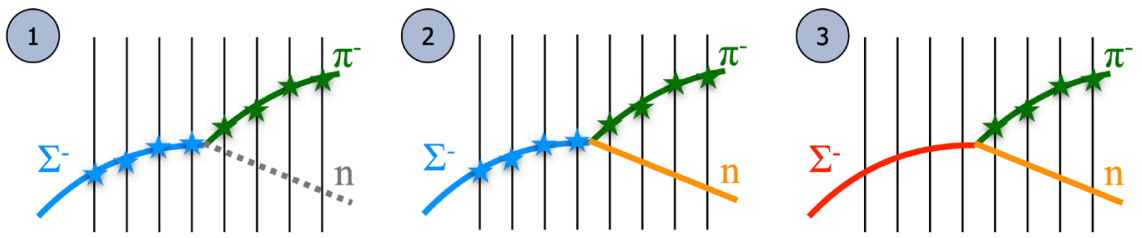

Figure 1. Three stages of reconstruction of a short-lived charged particle using the missing mass method

Therefore, the invariant mass of the daughter neutron can be found using the parameters of the registered mother $\Sigma^{-}$particle and the daughter pion:

$$
M_{n}=\left(P_{\Sigma^{-}}-P_{\pi^{-}}\right)^{2},
$$

where $P$ is a 4 -vector $P=\left(E, p_{x}, p_{y}, p_{z}\right)$.

A specific feature of this type of decays is that the short-lived mother track usually cannot be identified, making it impossible to directly calculate the missing neutron mass. However, 
the momentum of the mother particle $\Sigma^{-}$, unlike its mass, can be determined from the curvature of the trajectory. Therefore, we can assume and apply the hypothesis about the mass of the mother track, and then look at the resulting neutron mass spectrum.

Two cases are possible here:

1. if the mother particle was indeed $\Sigma^{-}$, there will be a peak in the neutron mass region $(939 \mathrm{MeV})$

2. if the hypothesis is not true, such cases will give a contribution to the continuous combinatorial background, created by the contribution of other particles and ghosts, in the neutron mass spectrum.

Then, to get the mass spectrum for $\Sigma^{-}$, we can use the mass of the neutron and calculate the state vector of the mother particle $\Sigma^{-}$from the two daughter tracks. The peak from the neutron mass spectrum will form a peak in the $\Sigma^{-}$mass region $(1.2 \mathrm{GeV})$, and the background will keep its continuous form and be turned into the background of the $\Sigma^{-}$mass spectrum.

Thus, the reconstruction of a short-lived charged particle using the missing mass method is performed in three steps (Fig. 1):

1. reconstruction of tracks of a charged mother particle $\left(\Sigma^{-}\right)$and its charged daughter particle $\left(\pi^{-}\right)$in the tracking system;

2. reconstruction of parameters of the neutral daughter particle $(n)$ by the parameters of the mother particle and the charged daughter;

3. reconstruction of the $\Sigma^{-}$mass spectrum using the charged particle and the obtained neutral daughter.

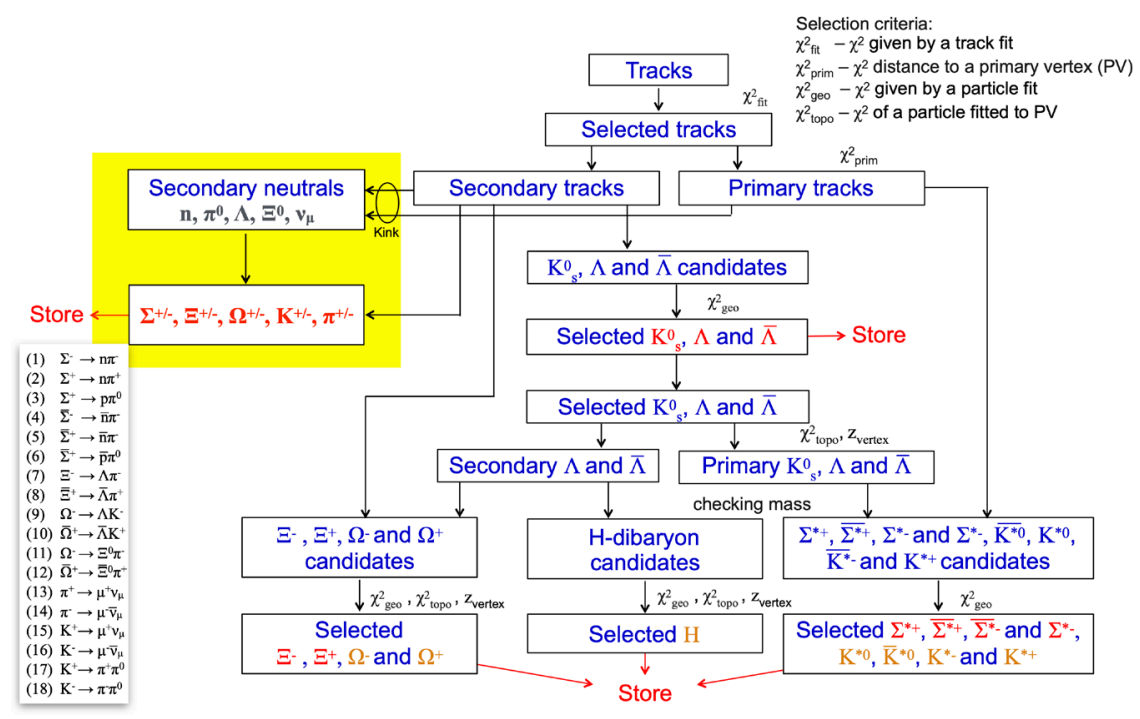

Figure 2. A simplified block diagram of the implementation of the missing mass method in the KF Particle Finder package

The method is implemented as an extension of the KF Particle Finder [2, 5] package, which is now actively used to search and reconstruct short-lived particles, both online and 
offline, in major heavy ion experiments such as CBM (FAIR/GSI), ALICE (CERN) and STAR (BNL).

All found tracks are given as input arguments, with secondary tracks - with PIDs, and primary tracks - without PIDs. If a secondary track is not identified for some reason, it is treated as a pion, the most common particle. For ease of processing, the secondary tracks are initially sorted in the following order: muons, pions, kaons, and protons. The method combines all primary tracks with the corresponding secondary tracks depending on the current hypothesis, e.g. all $\pi^{-}$in the case of $\Sigma^{-}$, or all $K^{-}$in the case of $\Omega^{-}$.

The KF Particle Finder package has now added a search for 18 decays with a neutral daughter particle (Fig. 2).

Before calculating the parameters of a neutral particle two main conditions are checked:

1. the first measurement of the charged daughter particle is farther away than the last measurement of the mother particle.

2. the energy of the primary particle is greater than the energy of the secondary particle. For the mother track, the energy is calculated according to the current hypothesis.

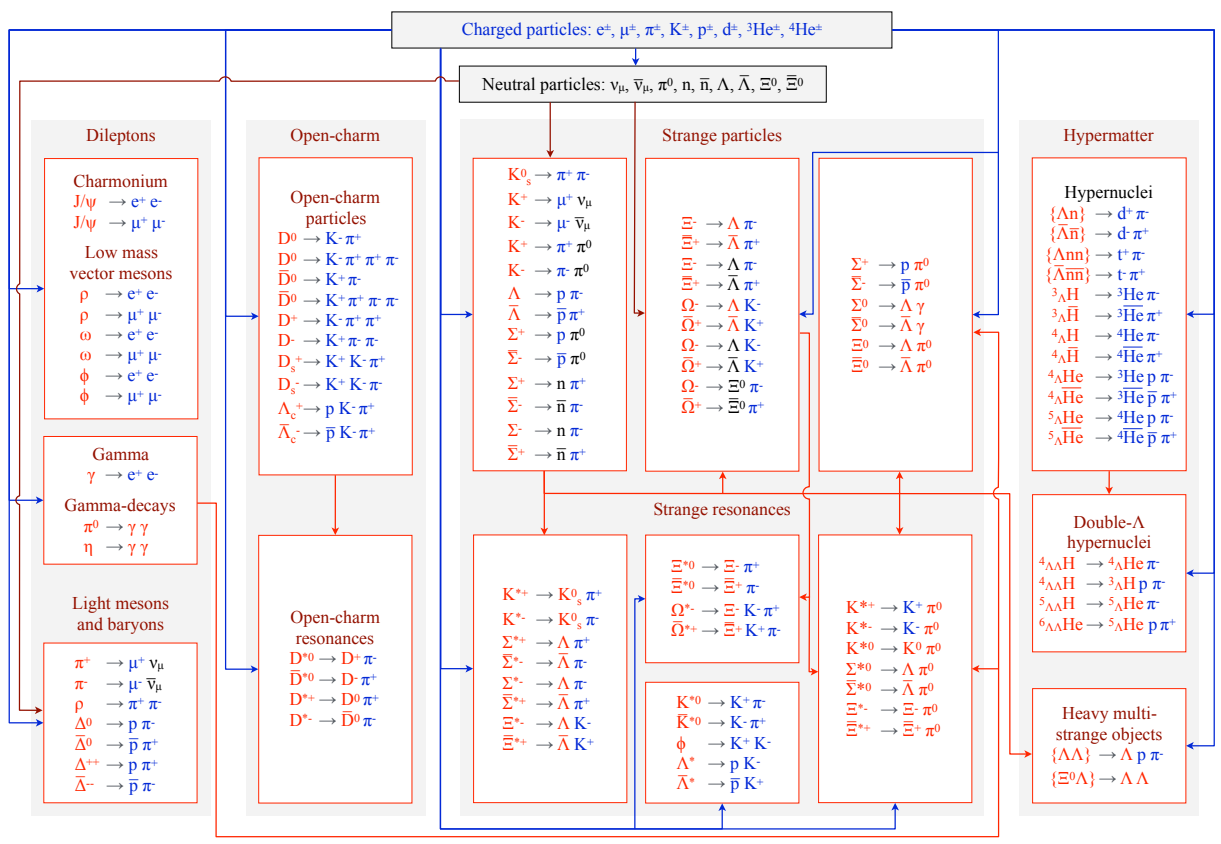

Figure 3. Block diagram of the reconstruction of short-lived particles in the KF Particle Finder package [5]

If both conditions are satisfied, the Kalman filter is applied to calculate the parameters of the neutral daughter particle at the point of their closest approach. Then, checking the quality of the fit, or more precisely, checking $\chi^{2}$, the method discards cases of non-overlapping tracks, and also checks the decay point between the two reconstructed tracks. It also allows the decay topology to require that the decay point be located after the last hit of the mother track and before the first hit of the daughter track. This requirement on the position of the reconstructed decay point suppresses significant part of the combinatorial background. 
These requirements, as well as some other conditions, have also been added to the KF Particle Finder package, which is now capable of finding up to 200 different decay channels, both offline and online (Fig. 3).

Once the candidates are found in the search for short-lived particles, there is competition among them to ensure that the daughter particles participate in only one, the most probable, decay channel. As an example, the decays $K_{s}^{0} \rightarrow \pi^{+} \pi^{-}$and $\Lambda \rightarrow p \pi^{-}$can be given, when in both decays one of the daughter particles is $\pi^{-}$.

The purpose of such a competition procedure is to allow the daughter pion to participate in only one decay. The application of such a competition procedure in the CBM experiment to simulated events at $25 \mathrm{GeV} / \mathrm{n}$, along with other measures, significantly improves the quality of the reconstruction of short-lived particles, in particular, the signal to background ratio. For example, after applying this final phase of analysis in the $K_{s}^{0}$ decay the signal to background ratio increased from 2.51 to 190 , and in the $\Lambda$ decay — from 8.15 to 269 .

Within the KF Particle Finder package, two approaches have been implemented to perform competition between particle candidates with possible common daughter particles: the deterministic approach and the artificial neural network approach.

In the deterministic approach the reconstruction quality of such competing candidate particles is compared and on the basis of certain criteria, such as $\chi^{2}$-value and closeness to the table mass value, the best particle is chosen.

In the artificial neural network approach, similar to its other applications [6-8] to optimize and select data, the network is first trained on the simulated data and then selects the best particles on its own.

Both approaches show almost identical results [9], so the choice of one for data processing is purely practical. Although we expect that the use of the artificial neural network may have some advantages when working on graphics cards.

The missing mass method has been repeatedly and successfully tested within the KF Particle Finder package on simulated data of the CBM experiment, demonstrating its value to the physics program of the experiment [10].

\section{Application of the method in the STAR experiment}

As part of the FAIR Phase-0 program [3, 4], the missing mass method has been successfully tested on pion and kaon decay channels offline on real STAR data from 2016 [11] and 2020 [12] data sets.

Here we present the results of applying the missing mass method to search for pion $\left(\pi^{ \pm} \rightarrow\right.$ $\left.\mu^{ \pm} v\right)$ and kaon $\left(K^{ \pm} \rightarrow \mu^{ \pm} v\right.$ and $\left.K^{ \pm} \rightarrow \pi^{ \pm} \pi^{0}\right)$ decays in real data of STAR obtained in May 2021 within the beam energy scan program BES-II and reconstructed online running the express data production and analysis chain.

The second phase [13] of the Beam Energy Scan at RHIC, BES-II (2019-2021), aims to investigate in $\mathrm{Au}+\mathrm{Au}$ collisions with precision measurements the high baryon density region of the QCD phase diagram [14]. The physics program of BES-II research is a continuation and refinement of the BES-I program that took place in 2010-2014. Some of the key measurements are the net-proton kurtosis that could pinpoint the position of a critical point, the directed flow that might prove a softening of the EOS, and the chiral restoration in the dilepton channel.

A fixed-target program, which uses only one beam on an internal target, allows to reach higher $\mu_{B}$ with high luminosity compared to the collider mode. The internal target is a thin $\mathrm{Au}$ foil inserted in the beam pipe at a distance of $2 \mathrm{~m}$ from the nominal interaction point, and a single circulating beam is directed to interact with it. 
More accurate measurements in BES-II are possible due to the order of magnitude better statistics provided by the upgrade of the electron cooling system in the RHIC, as well as the upgrade of detectors, primarily the upgrade of the inner Time Projection Chamber (iTPC) [15] and the end-cap Time Of Flight (eTOF) [16].

The main goals of the iTPC upgrade, replacing all 24 previous inner sectors in the STAR TPC with new ones, are wider rapidity coverage, lower $p_{T}$ cutoff, better momentum resolution, and better $d E / d x$ resolution. This increases $|\eta|$ coverage from 1 to 1.5 , and the lower threshold for the $p_{T}$ reconstructed particle momentum in the iTPC is reduced from $125 \mathrm{MeV} / \mathrm{c}$ to $60 \mathrm{MeV} / \mathrm{c}$.

The eTOF upgrade was done as part of the FAIR Phase- 0 program by the CBM and STAR collaborations to install in STAR $10 \%$ of the full TOF detector system of the CBM experiment. The eTOF detector covers a pseudorapidity range of -1.1 to -1.6 , i.e., only one side of the STAR detector. Combined with the iTPC upgrade, the eTOF upgrade will provide additional forward particle identification capabilities in STAR, greatly improving the fixed-target program at BES-II.
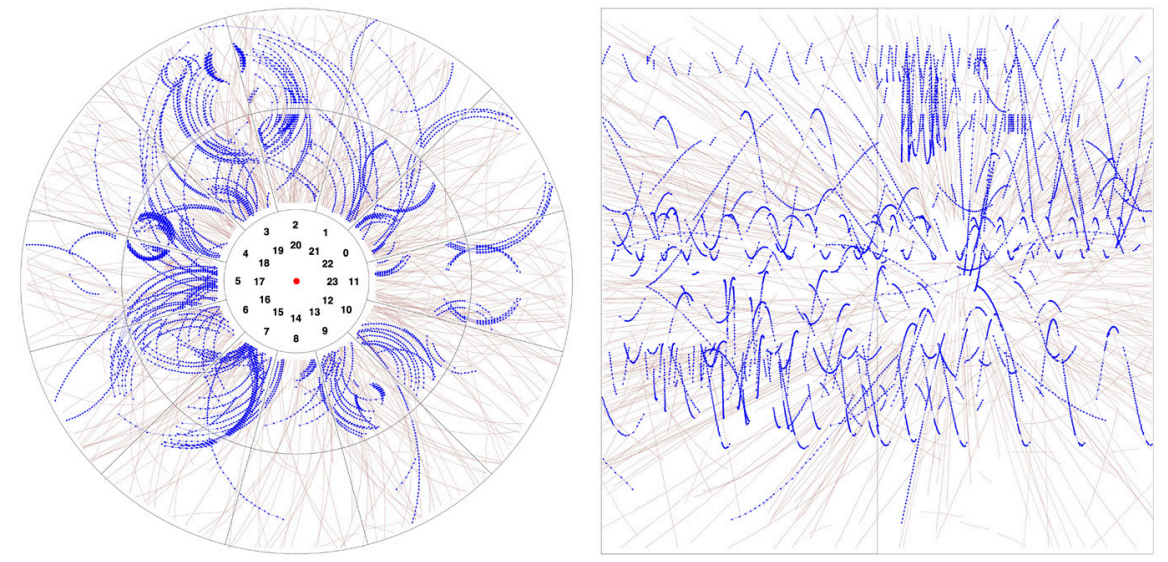

Figure 4. An example of the Cellular Automaton track finder on a reconstructed event with 30 lowmomentum returning tracks and other 346 reconstructed tracks, which are shown in background color. The front view to the left additionally shows the middle circle, which indicates the separation of the TPC detector into the inner part (iTPC) and the outer part.

The upgrade of iTPC and eTOF demanded a substantial modification of the track reconstruction algorithm based on the Cellular Automaton.

For instance, the reconstruction efficiency of low-momentum particles, including lowenergy particles that make several turns inside the TPC, the so-called loopers, was increased in accordance with the reconstruction requirements in the extended iTPC acceptance as well as in the forward direction of eTOF. The difficulty of reconstructing such particles is due both to their typical difficulties, such as long produced clusters, substantial multiple scattering and energy losses, but also to the fact that the track model itself at its core implies movement of particles from the detector center towards the outer PID detectors.

The Figure 4 shows an event display with an example of a simulated event with reconstructed 30 returning tracks. In this event, all phases of the returning trajectories are combined at the merging stage into long tracks, which, however, is not shown in the figure due to the technical requirement to follow the default track model. The other 346 tracks reconstructed in the standard way are also shown in the figure in the background color for completeness. 
Additionally, on the front view, the middle circle shows the separation of the TPC detector into the inner part (iTPC) and the outer part.

The launch of the iTPC and eTOF detectors in STAR, as well as the operation online in the fixed-target mode, demanded a substantial adaptation of the KF Particle Finder as well.

The new version uses now a more accurate and faster mathematical apparatus. For example, all the main calculations are done with $6 \times 6$ matrices instead of the former $7 x 7$. This was made possible by removing the energy from the state vector and the covariance matrix, and by calculating the energy and mass separately at the final stage. The parameters of all decay particles are now found in one iteration of the Kalman filter instead of the former two. In addition, this allowed us to abandon the very resource-intensive mass-constrained method, which balanced the parameters of the particles in some parts of the calculations.

Another significant change concerned the method of finding the secondary vertex. The decay point of the charged particle, or its secondary vertex, is now found in two steps at once at the initial stage and does not require refinement and recalculation later, which is mathematically more accurate, whereas previously the coordinates of the charged daughter particle participated in the calculation of the vertex position twice, which improperly overestimated their weight, that is significant especially for low-momentum particles.

Another part of the improvement is made to clear the background from particles knocked out of the detector material or in the region of the beam pipe and the separator between the inner and outer parts of TPC.

These modifications in the KF Particle Finder and its part of the missing mass method resulted in a significant improvement in the efficiency of the search for short-lived particles. Thus, in the pion decay channels $\pi^{ \pm} \rightarrow \mu^{ \pm} v$ the signal increased by a factor of 40 , and the significance increased by a factor of 7 . In the kaon decay channels $K^{ \pm} \rightarrow \mu^{ \pm} v$ and $K^{ \pm} \rightarrow \pi^{ \pm} \pi^{0}$, the signal increased by a factor of 2 , and the significance increased by a factor of 1.5 .

The rich physics program of BES-II also required fast data processing and real-time preliminary physics analysis in order to be able not only to monitor the quality of the acquired data, but also to evaluate the achievement of the expected physical result during data acquisition, and to make corrections to the accelerator operation plan, if necessary.

To this end, the functionality of the High Level Trigger was significantly extended (Fig. 5) when, in addition to the standard trigger tasks of selecting events to be stored on the media, a so-called express chain of data processing and analysis was created. Within the express chain, all necessary tasks, namely online calibration, reconstruction and analysis of data, were performed on the free resources of the HLT cluster.

Express calibration was performed in real time using the same algorithms as the standard offline data processing. Thus, the online data calibration was actually the first step in the calibration process, which reduced the time to achieve the final calibration. Track reconstruction was performed using the Cellular Automaton based track finder, which was a more advanced and faster version of the standard Cellular Automaton based track finder used in the standard offline data processing. Physics analysis of the data was also performed with the more advanced KF Particle Finder package compared to the offline data processing package.

Note that the express production and data analysis were performed with incomplete detector calibration and alignment, which posed additional challenges to the reconstruction and analysis algorithms.

We should also note that the express production was performed on the HLT cluster under conditions of limited computing resources and disk space so as not to interfere with the traditional HLT tasks of analyzing and selecting heavy-ion collisions in real time. Therefore, the express chain did not process and analyze all of the data, but only some of it, depending on the intensity of the data acquisition, but on average about $30 \%$. If necessary, it was possible 


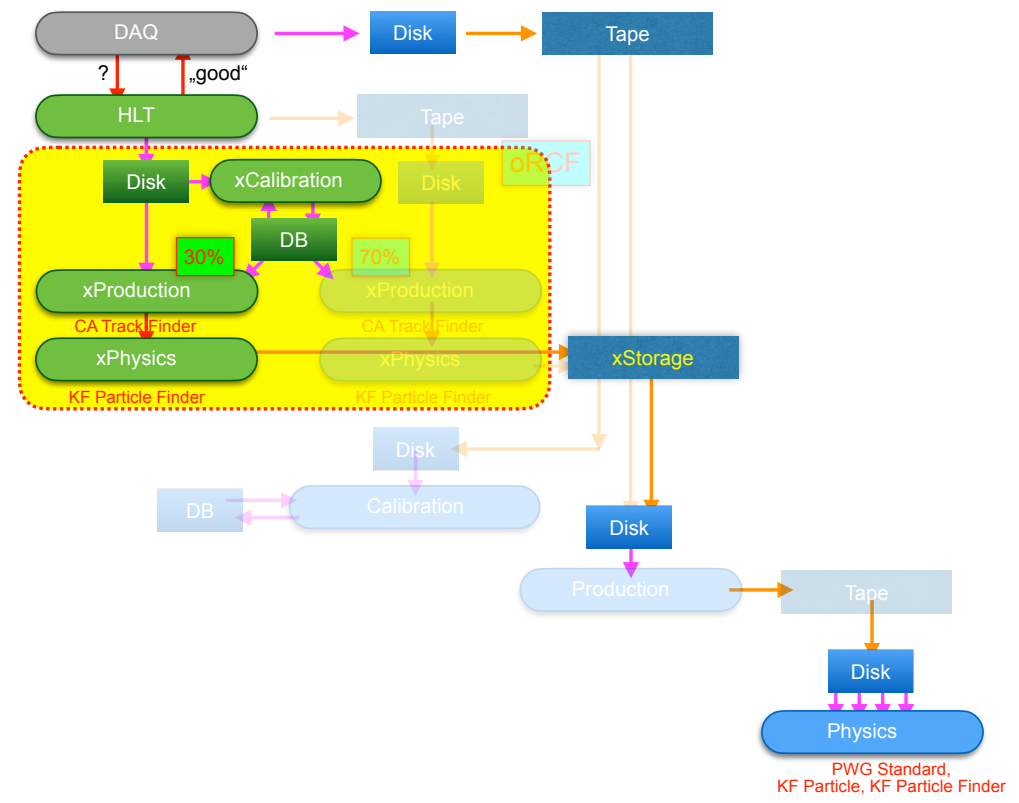

Figure 5. Scheme of online data analysis in their express processing on the HLT cluster of the STAR experiment

to involve additional computer resources of the RCF farm, where fast off-line data production was also carried out in real time in order to monitor the operation of detector systems in the process of data acquisition.

The full cycle of express processing of new data from their acquisition to obtaining physics results usually took about an hour of running on the HLT cluster, but if it became necessary to study in more detail the acquired data or the detector operation, additional processing cycles were carried out, which could take up to one day.

The data processed in the express chain were copied as picoDST files to the RCF farm for possible further analysis by different physics groups within the standard offline analysis chain. However, we should note that neither express data production nor fast offline data production interfered with the standard offline data production chain.

As a result, the need for fast data processing and analysis has led to the development of faster and more advanced algorithms, which will later be incorporated into the framework for standard offline data processing in the STAR experiment.

In addition, the use of similar, and often the same algorithms in both online and offline data processing create a bridge between online and offline processing chains, which allows us to consider the express production and data analysis as the first stage of the standard data production and analysis. Moreover, the results of data analysis in the express chain can serve as a reliable estimate of the final analysis, which will be obtained after the final calibration is achieved and the standard offline data production is performed.

Here we present the results of applying the missing mass method within the express chain to 9M minimum bias AuAu data acquired on May 1-2, 2021 at a beam energy of $3.85 \mathrm{GeV} / \mathrm{n}$ in the fixed-target mode (Fig. 6). The method was used to find $\pi^{+} \rightarrow \mu^{+} v_{\mu}, \pi^{-} \rightarrow \mu^{-} \bar{v}_{\mu}$, $K^{+} \rightarrow \mu^{+} v_{\mu}, K^{-} \rightarrow \mu^{-} \bar{v}_{\mu}, K^{+} \rightarrow \pi^{+} \pi^{0}$ and $K^{-} \rightarrow \pi^{-} \pi^{0}$ decays. 

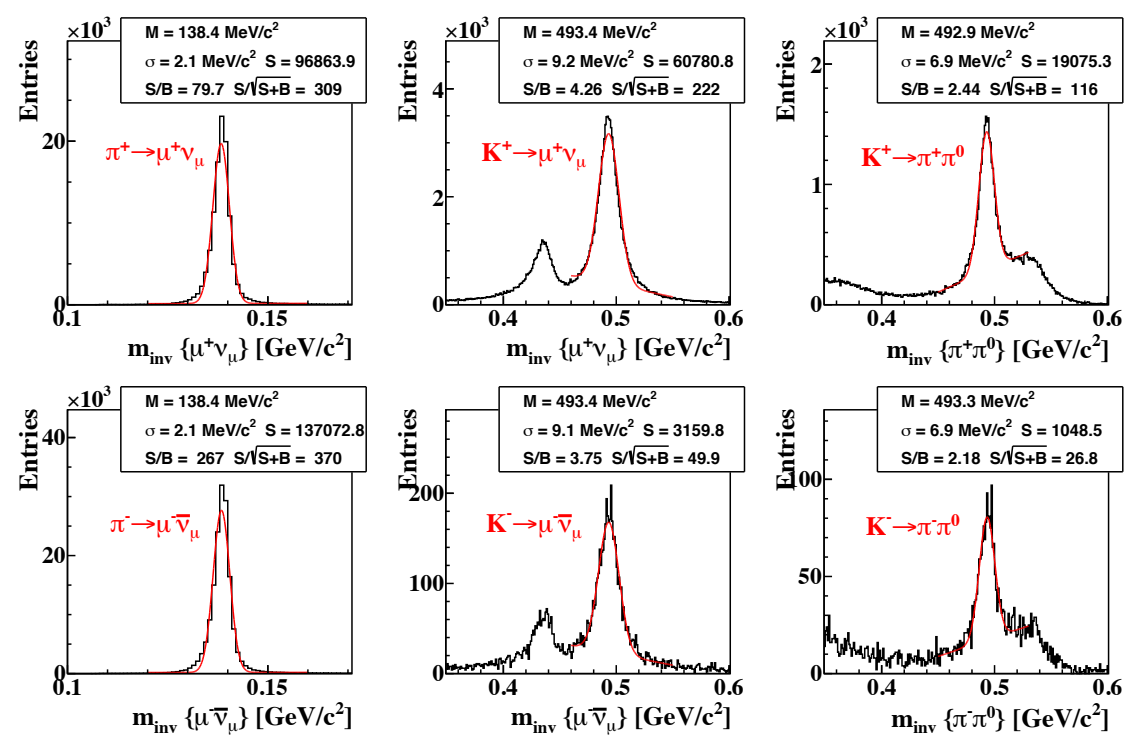

Figure 6. Results for $\pi^{ \pm} \rightarrow \mu^{ \pm} v, K^{ \pm} \rightarrow \mu^{ \pm} v$ and $K^{ \pm} \rightarrow \pi^{ \pm} \pi^{0}$ spectra obtained by the missing mass method using 9M minimum bias AuAu fixed-target collisions at a beam energy $3.85 \mathrm{GeV} / \mathrm{n}$, collected May 1-2, 2021, and processed online within the express production chain on the STAR HLT cluster

Application of the method to real data shows very good results with a high signal-tobackground ratio and a large value of significance. In the case of pion decays $\pi^{ \pm} \rightarrow \mu^{ \pm} v$ it was possible to obtain an essentially pure signal with almost no background. In the kaon decays $K^{ \pm} \rightarrow \mu^{ \pm} v$, a second peak is observed, which is the result of misidentification of $\pi$ as $\mu$.

Note again that these results were obtained during the online analysis in the express production chain on the HLT cluster while the detector system was still undergoing calibration and alignment. With standard offline data processing, the results will be improved due to the full calibration of all sub-detector systems of the STAR setup being achieved.

\section{Conclusion}

The KF Particle Finder package, originally developed for the First Level Event Selection (FLES) in the CBM experiment (FAIR/GSI, Germany), has been successfully applied within the FAIR Phase-0 program for online data analysis of the STAR experiment (BNL, USA).

The missing mass method makes it possible to significantly extend the physics programs of experiments in both high-energy and heavy-ion physics. The method is implemented as part of the KF Particle Finder package for reconstruction of short-lived particles with a neutral daughter particle.

Like the whole package, its part that implements the missing mass method has shown high efficiency and reliability when working in real time on the HLT cluster in the express data production and analysis chain. The advantage of the express chain is that it provides the shortest way from data acquisition to its physics analysis.

The results of the online application of the missing mass method to real data show that it provides data reconstruction with high signal to background ratio and significance. And 
the modification of the implementation of the method has resulted in an increase in the speed of the method and a significant improvement in the efficiency of the search for short-lived particles.

The successful application of the KF Particle Finder package to real data in the STAR experiment at low beam energies in the fixed target mode is of great importance also for the CBM experiment, where the package will be used to search for short-lived particles at similar beam energies, but at much higher up to $10^{7}$ interaction rates.

\section{References}

[1] I. Kisel, "Event reconstruction in the CBM experiment", Nucl. Instr. Meth. A 566 (2006) 85.

[2] I. Kisel, I. Kulakov, and M. Zyzak, "Standalone First Level Event Selection Package for the CBM Experiment", IEEE Trans. on Nucl. Science, 60, 5, (2013) 3703.

[3] E. Lavrik (on behalf of CBM Collaboration), "Compressed Baryonic Matter Experiment at FAIR", AIP Conference Proceedings 2163, 030009 (2019).

[4] I. Kisel (for CBM and STAR Collaborations), "Real-Time Event Reconstruction and Analysis in CBM and STAR Experiments", Journal of Physics: Conference Series 1602 (2020) 012006.

[5] M. Zyzak, "Online Selection of Short-Lived Particles on Many-Core Computer Architectures in the CBM Experiment at FAIR", Dissertation thesis, Goethe university, Frankfurt am Main (2016).

[6] Development of machine- and deep-learning tools for data analysis and detector design, https://www.erum-data-idt.de/institutes/fias/

[7] Artificial neural networks for optimisation in online marketing, https://ann2thrive.com/

[8] F. Sergeev, E. Bratkovskaya, I. Kisel and I. Vassiliev, "Deep learning for quark gluon plasma detection in the CBM experiment", International Journal of Modern Physics A, Vol. 35, No. 33 (2020) 2043002.

[9] A. Banerjee, I. Kisel and M. Zyzak, "Artificial neural network for identification of short-lived particles in the CBM experiment", International Journal of Modern Physics A, Vol. 35, No. 33 (2020) 2043003.

[10] V. Akishina, I. Kisel, P. Kisel, P. Senger, I. Vassiliev, and M. Zyzak, "Reconstruction of Particles Produced at Different Stages of Heavy Ion Collision in the CBM Experiment at FAIR”, Poster, Quark Matter 2017, 5-11 February 2017, Chicago, USA.

[11] Y. Fisyak, I. Kisel, P. Kisel, P. Senger, I. Vassiliev, and M. Zyzak, "Applying the Missing Mass Method to the STAR at RHIC data", CBM Progress Report 2017, GSI, Darmstadt, doi:10.15120/GSI-2018-00485, (2018) p. 197.

[12] Y. Fisyak, V. Ivanov, H. Ke, I. Kisel, P. Kisel, G. Kozlov, A. Tang, and I. Vassiliev, "Application of the Missing Mass Method in STAR BES-II fixed-target program", CBM Progress Report 2020, GSI, Darmstadt, doi:10.15120/GSI-2021-00421, (2021) p. 223.

[13] C. Yang (for the STAR Collaboration), "The STAR beam energy scan phase II physics and upgrades", Nuclear Physics A 967 (2017) 800.

[14] STAR Collaboration, "Studying the Phase Diagram of QCD Matter at RHIC", STAR Note 598, Mar. 28, 2014, https://drupal.star.bnl.gov/STAR/starnotes/public/sn0598

[15] STAR Collaboration, "Technical Design Report for the iTPC Upgrade", STAR Note 644, Nov. 30, 2015, https://drupal.star.bnl.gov/STAR/starnotes/public/sn0644

[16] I. Deppner and N. Herrmann, "The FAIR Phase 0 program of the CBM TOF", Journal of Instrumentation 15 (2020) C10030. 